\title{
Extranodal Disease
}

National Cancer Institute

\section{Source}

National Cancer Institute. Extranodal Disease. NCI Thesaurus. Code C39695.

A clinical finding that refers to the spread or absence of lymphoid cancer in anatomic sites or systems other than the original lymph node site of growth. 